\title{
Mass Spectrometric Strategy for the Characterization of Lipooligosaccharides from Neisseria gonorrhoeae 302 Using FTICR
}

\author{
Michael D. Leavell and Julie A. Leary \\ University of California, Berkeley, California, USA \\ Ryohei Yamasaki \\ Tottori University, Tottori, Japan
}

\begin{abstract}
The lipooligosaccharides (LOS) of Neisseria gonorrhoeae 302 were profiled using Fourier transform ion cyclotron resonance mass spectrometry (FTICR-MS). Using techniques developed in this laboratory, the topology and some of the linkages of the LOS were determined. Mass spectrometric analysis in the negative ion mode yielded a glycoform of the composition: $\mathrm{Hex}_{3} \mathrm{Hep}_{2} \mathrm{Hxn}_{1} \mathrm{PEA}_{1} \mathrm{KDO}_{2}$ DPLA. The composition was confirmed through exact mass measurements, which showed only a 2 ppm error between the exact mass and theoretical mass. Although the core structure has been postulated previously, the positioning of the three hexose moieties were in question for this particular strain of $N$. gonorrhoeae. Topology assignment was performed through collision-induced dissociation analysis of the O-deacylated glycoform in the negative ion mode followed by submission to the saccharide topology analysis tool (STAT) computer program, which confirmed the topology assignment. It was found that the three hexoses were added to the Hep[I] of the conserved core of $N$. gonorrhoeae in a linear fashion, while Hep[II] remains unbranched. Linkage position analysis was performed through application of a mild acid hydrolysis technique followed by collisioninduced dissociation of the sodiated precursor ions, yielding a $1 \rightarrow 4$ linkage between the terminating and penultimate hexoses. (J Am Soc Mass Spectrom 2002, 13, 571-576) (c) 2002 American Society for Mass Spectrometry
\end{abstract}

$\mathrm{N}$ eisseria gonorrhoeae is one of the major sexually transmitted diseases worldwide accounting for $\sim 20 \%$ of all curable sexually transmitted disease (STD) infections [1, 2]. Untreated, gonococcal infections can result in complications such as blindness, infertility, arthritis, endocarditis, and meningitis [1, 2]. Furthermore, gonococcal infections have been shown to facilitate the spread of HIV [2]. Given the widespread occurrence and the potentially serious health effects of gonococcal infection, development of several vaccines has been attempted. These attempts utilized the pilus, porin, or the opacity proteins on the bacterial cell surfaces. Unfortunately, vaccines of only a limited protective immune response have been found using these moieties [3]. In a further effort to find a vaccine against Neisseria gonorrhoeae, focus has turned to the bacterial lipooligosaccharides (LOS).

Bacterial LOS are the principal components of the bacterial cell surface. Given their location, it is not

Published online April 2, 2002

Address reprint request to Dr. J. A. Leary, Department of Chemistry, University of California, Berkeley, CA 94720, USA. E-mail: leary@socrates.berkeley.edu surprising that the LOS have been found to activate the immune response of the host. Activation occurs through interaction of the LOS with the host's cell membranes and circulating glycoproteins. Immunochemical studies have shown that LOS epitopes mimic human glycolipids, which are precursors to the blood group antigens [4-6]. Also, bacterial LOS have been shown to be sialylated by the host in vivo [6]. Given these facts, and the fact that phase variation has been observed at different stages of bacterial infection [6], it is believed that the LOS allow the bacteria to evade the host's defense mechanisms.

Over the years, several methods of carbohydrate sequencing have emerged, however, there is no single method that may be applied to the determination of carbohydrate structure. Several reasons make carbohydrates an especially difficult class of compounds to analyze; these include the many different linkage positions that are possible within a carbohydrate, the branching positions, and the fact that the anomericity of each glycosidic bond and the stereochemistry of each monosaccharide may vary. Mass spectrometry (MS) has proven itself to be an attractive alternative to other methods of carbohydrate sequencing. This is due to the speed of MS, its sensitivity, and the ability to use gas 
phase isolation, which obviates the need for chromatography [7-10]. In our laboratory, we have developed several methods for the determination of carbohydrate structure [11-17]. These include methods for topology, linkage position, stereochemistry, and anomericity determination. In the study presented herein, several of these methods are used to analyze the structure of $N$. gonorrhoeae strain 302 . Initially, the glycoform population present on the cell surface of the bacteria was determined using exact mass measurements on a FTICR mass spectrometer. Subsequently, tandem mass spectrometry $\left(\mathrm{MS}^{\mathrm{n}}\right)$ was employed to determine the topology of the $\mathrm{Hex}_{3}$ glycoform (Hex: hexose) from the N. gonorrhoeae bacteria, and confirmed using the STAT computer program [17]. This program has the advantage of generating all structures that are possible from the observed collision-induced dissociation (CID) data, thus possible structures will not be missed. Although the core structure of $N$. gonorrhoeae has been postulated previously, it was the position and linkages of the three hexose moieties that were in question for this particular strain of N. gonorrhoeae. Finally, to determine the linkage position within the $\mathrm{Hex}_{3}$ glycoform, a mild acid hydrolysis technique was utilized to generate fragment ions with newly generated reducing ends [16]. This latter technique, combined with alkali metal coordination followed by CID, has been shown to yield linkage information within the oligosaccharide (OS) sample $[10-12,18]$.

\section{Experimental}

\section{Chemicals}

LOS isolated from N. gonorrhoeae 302 were obtained from Dr. Ryohei Yamasaki, Department of Biochemistry and Biotechnology, Tottori University (Tottori, Japan). Acetic acid was purchased from Fisher (Pittsburgh, PA). HPLC grade acetonitrile and water were purchased from Sigma (St. Louis, MO). All other reagents and solvents were of reagent grade, and used as received.

\section{Chemical Treatments of LOS}

The LOS from Neisseria gonorrhoeae 302 was purified using the hot phenol extraction method [19]. O-deacylation [20], dephosphorylation [20], lipid removal [20], and mild acid hydrolysis [16] were all performed as described previously.

\section{MS Analysis by FTICR}

FTICR experiments were performed on an APEX II FTICR mass spectrometer equipped with a $7 \mathrm{~T}$ actively shielded superconducting magnet (Bruker Daltonics, Billerica, MA). Ions were generated with an Analytica electrospray ionization (ESI) source (Analytica, Branford, CT) in either positive or negative ion mode. The generated ions were externally accumulated for $2 \mathrm{~s}$ in

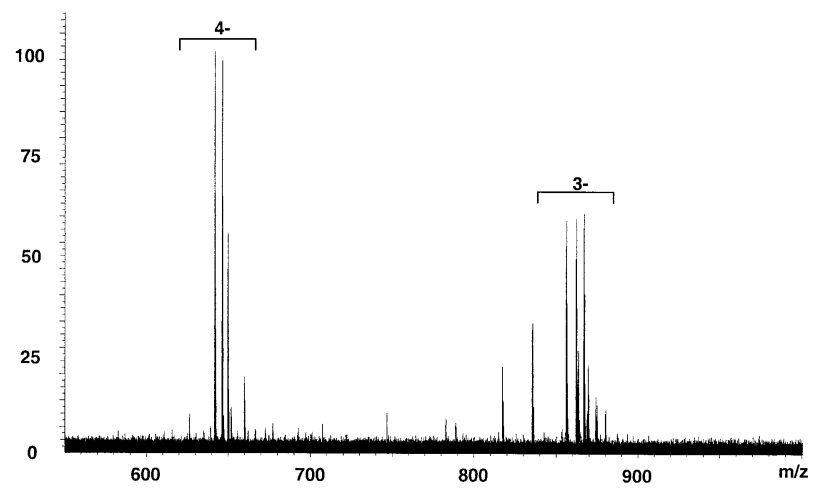

Figure 1. MS ${ }^{1}$ spectrum of the O-deacylated LOS sample from $N$. gonorrhoeae 302.

an Rf-only hexapole ion guide before being transferred to the ICR cell for mass analysis. Exact mass measurements were performed by using $\mathrm{NaI}$ as an external calibrant. $\mathrm{MS}^{\mathrm{n}}$ analysis was performed through isolation of the desired ion using correlated sweep, which is controlled using correlated harmonic excitation fields. Sustained off-resonance excitation (SORI) was applied for CID. A pressure of $\sim 2 \times 10^{-6}$ mbar of argon was introduced into the ultrahigh vacuum region, and the ion of interest was activated for $250 \mathrm{~ms}$ with a radiofrequency pulse at $500 \mathrm{~Hz}$ above its resonant frequency. Each spectrum is an average of 16-64 transients composed of $512 \mathrm{k}$ points acquired using a SGI workstation operating XMASS version 5.0.10.

\section{Results and Discussion}

To ascertain the glycoform population present on the surface of the $N$. gonorrhoeae bacteria, a MS ${ }^{1}$ spectrum was taken of the O-deacylated sample (Figure 1). Odeacylation removes portions of the acyl chains from the lipid A moiety, which increases the ionization efficiency of the sample. Both the triply- and the quadruply-charged ion populations were observed in the MS $^{1}$ spectrum. The ions at $\mathrm{m} / \mathrm{z} 646.2315$ and 861.9762 correspond to the $4^{-}$and the $3^{-}$charge states of a glycoform with the composition: O-DPLA $\mathrm{KDO}_{2} \mathrm{Hep}_{2}$ $\mathrm{Hxn}_{1} \mathrm{PEA}_{1} \mathrm{Hex}_{3}$ (notated: Hex 3 glycoform; O-DPLA: O-deacylated diphosphorylated lipid A, KDO: 2-keto3-deoxyoctulosonic acid, Hep: heptose, Hxn: N-Acetyl hexosamine, PEA: phosphoethanolamine, Hex: hexose). These ions were confirmed by exact mass measurements through external calibration, with less than 2 ppm error between the theoretical and measured masses. Other species were also observed in the MS spectrum. The ions at $\mathrm{m} / \mathrm{z} 641.7284$ and 855.9726 correspond to the $\left[\mathrm{Hex}_{3}-\mathrm{H}_{2} \mathrm{O}\right]$ glycoform for the $4^{-}$and $3^{-}$ charge states respectively. Loss of $\mathrm{HPO}_{3}$ from the $\mathrm{Hex}_{3}$ glycoform is observed at $\mathrm{m} / \mathrm{z}$ 835.3216; phosphate loss is common for phosphate containing saccharides. Other ions correspond to the sodiated adduct of the $\mathrm{Hex}_{3}$ glycoform $(\mathrm{m} / \mathrm{z}$ 869.3015) as well as cleavage of the lipid 
Table 1. Composition analysis of LOS from N. gonorrhoeae strain 302

\begin{tabular}{|c|c|c|c|c|}
\hline Composition & z & $\begin{array}{c}\text { Measured } \\
\mathrm{m} / \mathrm{z}\end{array}$ & $\begin{array}{c}\text { Theoretical } \\
\mathrm{m} / \mathrm{z}\end{array}$ & $\begin{array}{r}\text { Error } \\
\text { (ppm) }\end{array}$ \\
\hline DPLA KDO ${ }_{2} \mathrm{Hep}_{2} \mathrm{Hxn}_{1} \mathrm{PEA}_{1} \mathrm{Hex}_{3}-\mathrm{H}_{2} \mathrm{O}$ & 4- & 641.7284 & 641.7294 & 1.56 \\
\hline DPLA KDO ${ }_{2} \mathrm{Hep}_{2} \mathrm{Hxn}_{1} \mathrm{PEA}_{1} \mathrm{Hex}_{3}$ & 4- & 646.2315 & 646.2320 & 0.77 \\
\hline $\mathrm{KDO}_{2} \mathrm{Hep}_{2} \mathrm{Hxn}_{1} \mathrm{PEA}_{1} \mathrm{Hex}_{3}$ & $2-$ & 817.2365 & 817.2376 & 1.35 \\
\hline DPLA KDO ${ }_{2} \mathrm{Hep}_{2} \mathrm{Hxn}_{1} \mathrm{PEA}_{1} \mathrm{Hex}_{3}-\mathrm{HPO}_{3}$ & 3- & 835.3216 & 835.3230 & 1.58 \\
\hline DPLA KDO ${ }_{2} \mathrm{Hep}_{2} \mathrm{Hxn}_{1} \mathrm{PEA}_{1} \mathrm{Hex}_{3}-\mathrm{H}_{2} \mathrm{O}$ & 3- & 855.9726 & 855.9749 & 2.69 \\
\hline DPLA KDO ${ }_{2} \mathrm{Hep}_{2} \mathrm{Hxn}_{1} \mathrm{PEA}_{1} \mathrm{Hex}_{3}$ & 3- & 861.9762 & 861.9784 & 2.55 \\
\hline DPLA KDO $2 \mathrm{Hep}_{2} \mathrm{Hxn}_{1} \mathrm{PEA}_{1} \mathrm{Hex}_{3}+\mathrm{Na}$ & 3- & 869.3015 & 869.3058 & 4.95 \\
\hline
\end{tabular}

A moiety $(\mathrm{m} / \mathrm{z} 817.2365)$. A summary of the major ions observed is shown in Table 1.

It has been shown that the LOS generated by the $N$. gonorrhoeae bacteria have a commonly conserved core as shown in Figure 2 [6]. Previous studies suggest that the gonococci elongate their core by addition of hexoses to either Hep(I) or Hep(I) and Hep(II), however, to our knowledge branching of Hep(II) alone has not been observed. The core consists of a lipid A moiety, two Hep's, two KDO's, and one Hxn. The MS ${ }^{\mathrm{n}}$ experiments verify several of the features of the core. In this study, the location of the hexoses, and in some cases the linkage position, was derived solely from empirical evidence provided herein.

To determine the topology of the LOS sample, chemical treatments were performed to remove the lipid A moiety and the phosphate-containing groups. These treatments enhance fragmentation of the non-reducing end allowing more information to be gained for this portion of the oligosaccharide. Upon completion of these treatments, $\mathrm{MS}^{1}$ in the negative ion mode yielded an ion at $\mathrm{m} / \mathrm{z} 1310$, which corresponds to the composition: [KDO Hep $\left.\mathrm{Hxn}_{1} \mathrm{Hex}_{3}\right]^{1-}$. This composition is expected because the acetic acid treatment cleaves the glycosidic bonds adjacent to the acidic residues, removing the lipid A and the terminating KDO, while the HF

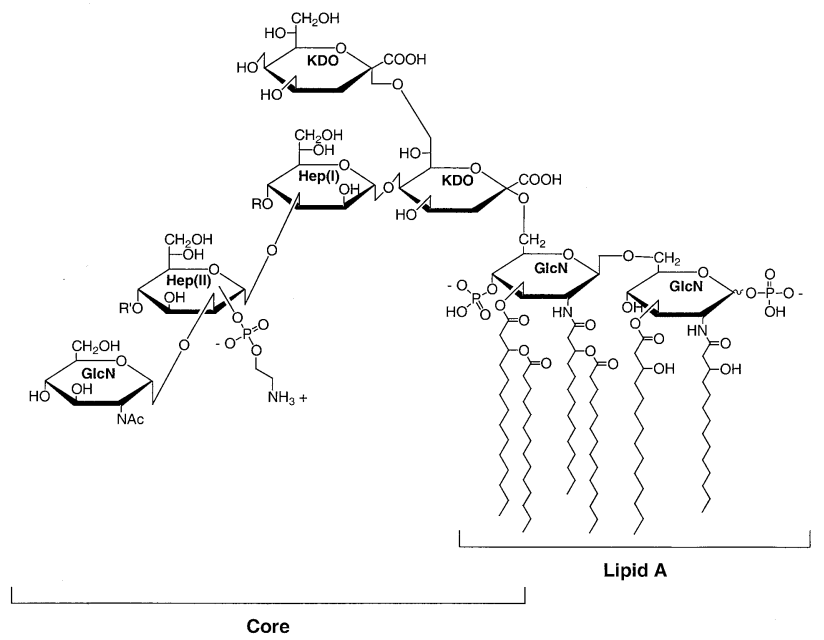

Figure 2. General structure of the N. gonorrhoeae LOS, where the Lipid A and the conserved core portions are shown. R and R' are the branching sites on the LOS. treatment cleaves the PEA. The linkage position of the PEA has been shown in previous studies to be linked at Hep(II). Employment of tandem mass spectrometry in the negative ion mode has been shown to yield ions that result from glycosidic cleavages [10, 20]; these cleavages allow determination of oligosaccharide topology.

$\mathrm{MS}^{2}$ of the ion at $m / z 1310$ yields (Figure 3 ) cross ring cleavages of the reducing $\mathrm{KDO}$ moiety as well as a glycosidic cleavage of the KDO from Hep(I). The two cross ring cleavages result in loss of $\mathrm{C}_{3} \mathrm{H}_{6} \mathrm{O}_{3} / \mathrm{H}_{2} \mathrm{O}\left({ }^{0,4} \mathrm{X}\right.$ cross ring cleavage followed by loss of $\mathrm{H}_{2} \mathrm{O}$ ) and $\mathrm{C}_{6} \mathrm{H}_{12} \mathrm{O}_{6}\left({ }^{2,4} \mathrm{~A}\right.$ cross ring cleavage) from the reducing $\mathrm{KDO}$. The glycosidic cleavage signifies a terminating KDO in accord with previous characterization of the $N$. gonorrhoeae core, as well as indicating either a $1 \rightarrow 4$ or a $1 \rightarrow 5$ linkage.

A plethora of low mass ions are also observed (Figure 4). These cleavages are from the non-reducing end of the OS and many of these correspond to glycosidic cleavage. The ions that yield topology information are found at $m / z 161,220,341,394,412$, and 503, these ions correspond to a composition of: $\operatorname{Hex}, \operatorname{Hxn}(R)$, $\operatorname{Hex}_{2}(\mathrm{R})$, Hxn-Hep, Hxn-Hep(R), and $\mathrm{Hex}_{3}(\mathrm{R})$, respectively, (where $\mathrm{R}$ corresponds to ions with a reducing end). Two other ions are observed at $m / z 262$ and 304. As is determined by their mass, these ions must contain a nitrogen [21]. Thus, these ions are believed to arise from cross ring cleavage of the Hep(II) moiety, resulting in ions containing the Hxn moiety that is known to exist in the core. Accordingly, $\mathrm{m} / \mathrm{z} 262$ corresponds to the

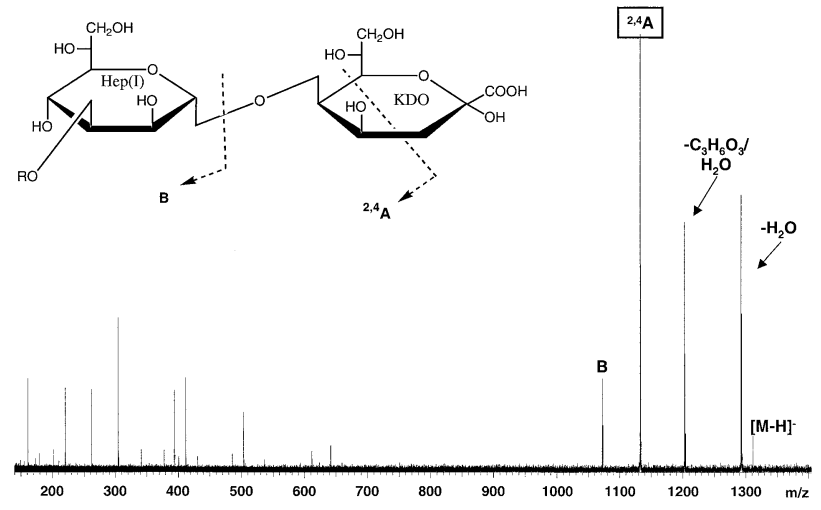

Figure 3. $\mathrm{MS}^{2}$ of the delipidated/dephosphorylated $\mathrm{Hex}_{3}$ glycoform from N. gonorrhoeae 302. 


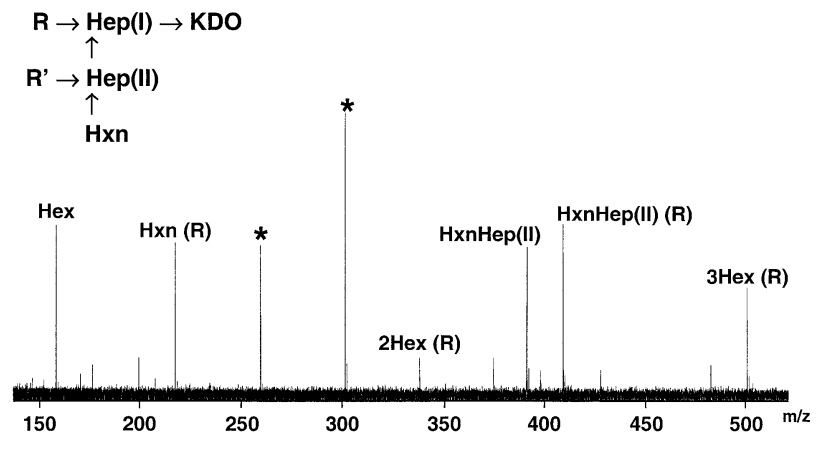

Figure 4. Low mass region of the $\mathrm{MS}^{2}$ spectrum shown in Figure 3. Ions that result from cross ring cleavage of Hep(I) are marked with an asterisk.

mass of $\mathrm{Hxn}+\mathrm{C}_{2} \mathrm{H}_{4} \mathrm{O}_{2}$, while $m / z 304$ corresponds to a composition of $\mathrm{Hxn}+\mathrm{C}_{4} \mathrm{H}_{6} \mathrm{O}_{3}$. The ions at $m / z 161$ and 220 correspond to a Hex and a $\operatorname{Hxn}(\mathrm{R})$ moiety, respectively. Since these saccharides are released as monomers, they must be in terminating locations on the oligosaccharide. The ions at $\mathrm{m} / \mathrm{z} 394$ and 412 are indicative of a Hep-Hxn disaccharide moiety. This suggests that the Hep and Hxn moieties are linked together somewhere in the OS structure. Is it not surprising that the terminating Hxn and the linked Hep-Hxn moieties are observed, since the core of $N$. gonorrhoeae is known to contain both of these epitopes [6].

Three other ions yield topology information of the non-reducing end. These are $m / z$ 161, 341, and 503, which correspond to a composition of $\operatorname{Hex}_{1}, \operatorname{Hex}_{2}(\mathrm{R})$, and $\operatorname{Hex}_{3}(\mathrm{R})$, respectively. Observation of the $\mathrm{Hex}_{3}$ moiety suggests that all three hexoses added to the non-reducing end must be linked. Furthermore, observation of the $\mathrm{Hex}_{2}$ moiety suggests that the three Hex's are linked together in a linear fashion, since this ion would not be possible for a branched structure.

$\mathrm{MS}^{3}$ of the ${ }^{2,4} \mathrm{~A}_{2}$ cross ring cleavage also yields a plethora of topology ions (Figure 5). Aside from the low mass ions observed at the previous stage of $\mathrm{MS}^{2}$, several glycosidic bond cleavages at the high mass end of the spectrum yields topology information (discussed below using our STAT program). In particular, loss of Hex,

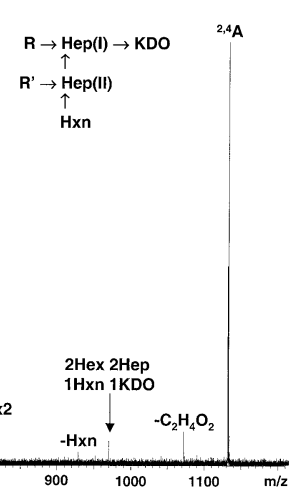

Figure 5. $\mathrm{MS}^{3}$ of the delipidated/dephosphorylated $\mathrm{Hex}_{3}$ glycoform from $N$. gonorrhoeae 302. Ions that result from cross ring cleavage of Hep(I) are marked with an asterisk.

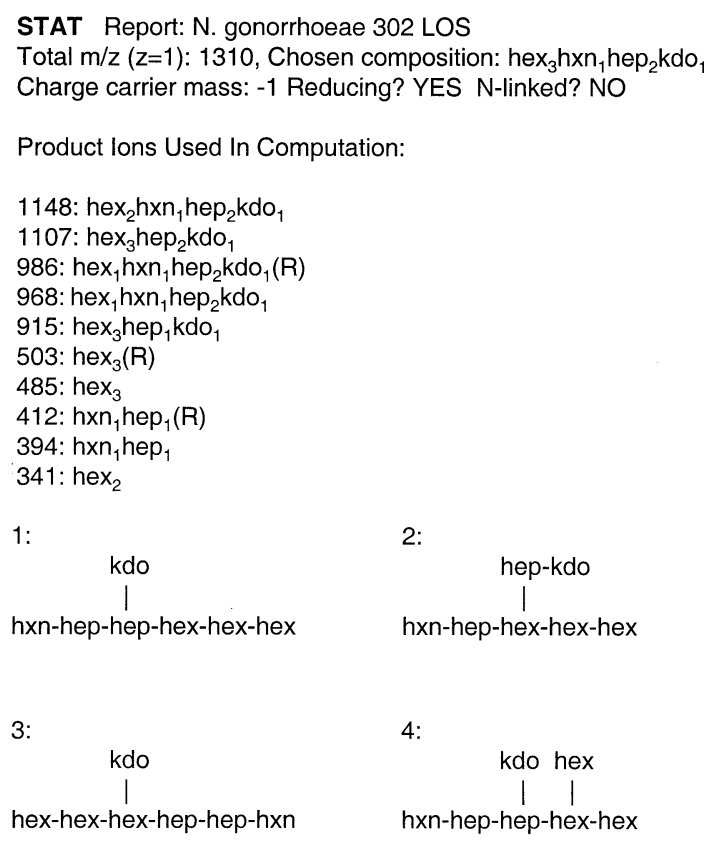

Figure 6. The generated output from the STAT computer program. Only structures (1) and (4) contain the conserved core of $N$. gonorrhoeae. The rank of each structure, as generated by STAT, is shown in the upper left corner of each structure.

Hxn, $\mathrm{Hex}_{2}$, and $\mathrm{Hex}_{3}$ is observed. These losses are consistent with the conclusions stated earlier, that the Hex's are linearly connected and a Hxn and a Hex are terminating. One other ion yields important information about the topology of the LOS, this ion is observed at $m / z 737$ and corresponds to a composition of $\mathrm{Hex}_{3} \mathrm{Hep}_{1}+\mathrm{C}_{2} \mathrm{H}_{4} \mathrm{O}_{2}$. Since this ion originated from the ${ }^{2,4} \mathrm{~A}_{2}$ cross ring cleavage precursor, it is likely that the addition of $\mathrm{C}_{2} \mathrm{H}_{4} \mathrm{O}_{2}$ was originally part of the reducing $\mathrm{KDO}$. This knowledge, taken in concert with the observation of the HxnHep(II) glycosidic cleavage without any additional Hex's, demonstrates that the three Hex's are branched from $\operatorname{Hep}(\mathrm{I})$, while Hep(II) remains unbranched.

Much of the data interpretation discussed above, regarding the topology, were extrapolated using the STAT program, developed previously in our laboratory [17]. STAT utilizes all of the $\mathrm{MS}^{\mathrm{n}}$ product ions to generate all possible topologies that could result from the observed product ions. Upon submission to STAT, 13 possible topologies were generated from the product ion data. The top four candidates are shown in Figure 6. The structures are ranked in the order of the fewest number of bond cleavages needed to produce the observed product ions. Upon examination of the 13 candidate structures, only hits (1) and (4) are given further consideration. This decision is based on the assumption that the Neisseria gonorrhoeae core is conserved in this particular strain. Thus, the strategy is to use all available biological and chemical information, in conjunction with STAT and the $\mathrm{MS}^{\mathrm{n}}$ profile, in order to obtain as much structural information as possible. As 


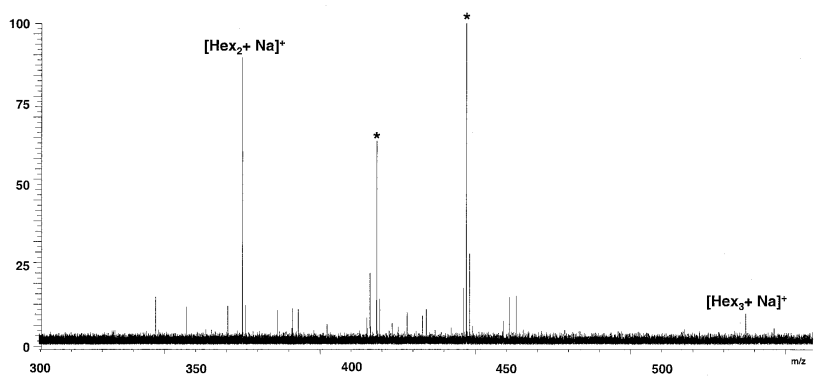

Figure 7. MS ${ }^{1}$ spectrum of the mild acid hydrolysis product from N. gonorrhoeae 302. Peaks originating from the resin are marked with an asterisk.

seen from Figure 6, the candidate topologies differ by the branching of the three Hex moieties. Since we know from the previous $\mathrm{MS}^{2}$ data (Figure 3) that the hexoses are unbranched, we propose that structure (1) is the topology for the $\mathrm{Hex}_{3}$ glycoform.

With the topology information in hand, the next step was to determine the linkages of the hexoses. To determine the linkage, a mild acid hydrolysis technique was utilized [16]. This technique was shown to produce smaller oligomers, which contain new reducing ends. An added advantage to the employment of this method is that acid reversion of glycosidic bonds is not observed, as is found under normal acid hydrolysis conditions. When these oligomers are cationized with alkali metals and subjected to CID, ions that are diagnostic to the glycosidic linkage position are observed. The MS ${ }^{1}$ spectrum of the hydrolysate from delipidated/dephosphorylated N. gonorrhoeae 302 is shown in Figure 7. Both the sodiated disaccharide and the sodiated trisaccharide moieties are observed. The disaccharide ion is much more abundant due to the susceptibility of the Hex-Hex glycosidic bond to acid hydrolysis as compared to the Hex-Hep glycosidic bond [16].

Upon CID of the $\left[\mathrm{Hex}_{2}+\mathrm{Na}\right]^{+}$ion $(\mathrm{m} / \mathrm{z} 365)$, two characteristic peaks of a $1 \rightarrow 4$ linkage are observed (Figure 8). These diagnostic ions have been reported
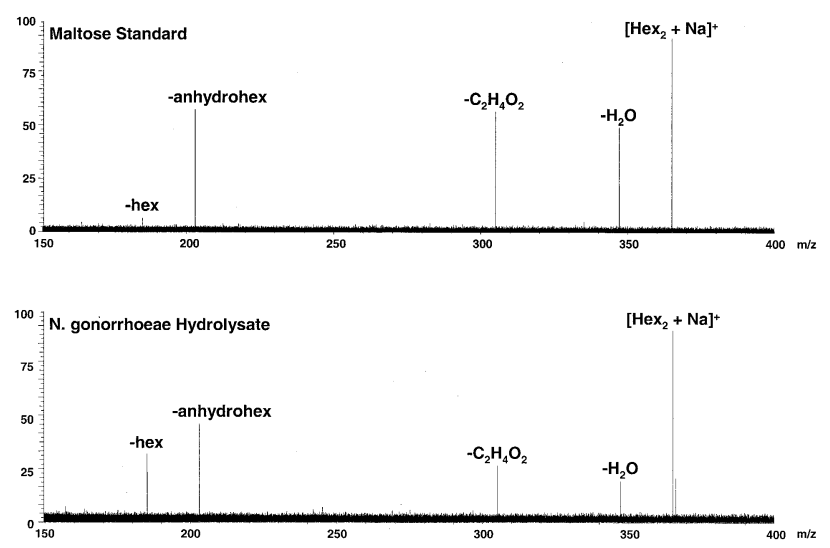

Figure 8. $\mathrm{MS}^{2}$ of the sodiated disaccharide ion from the mild acid hydrolysis of $N$. gonorrhoeae 302 and a maltose standard for comparison. Ions indicate a $1 \rightarrow 4$ linkage between the penultimate and terminating hexoses. before and are from losses of $\mathrm{H}_{2} \mathrm{O}$ and $\mathrm{C}_{2} \mathrm{H}_{4} \mathrm{O}_{2}[16,18]$. To confirm this data, maltose was subjected to CID under the same conditions as the $N$. gonorrhoeae hydrolysate. A comparison between these spectra are shown in Figure 8, thus confirming the $1 \rightarrow 4$ linkage between the terminating and penultimate hexoses [16, 18]. Unfortunately, the linkage between the penultimate and the hexose attached to heptose(I) could not be determined, because of the inability to generate abundant sodiated species. This is due to the difficulty hydrolyzing the Hex-Hep bond [16].

\section{Conclusions}

The LOS from N. gonorrhoeae strain 302 were analyzed. In the $\mathrm{MS}^{1}$ spectrum of the O-deacylated sample, one glycoform was found. Using exact mass measurements the composition was shown to be: $\mathrm{Hex}_{3} \mathrm{Hep}_{2} \mathrm{Hxn}_{1}$ $\mathrm{PEA}_{1} \mathrm{KDO}_{2}$ lipid A. The molecular ion exhibited only a 2 ppm error between the exact mass and the theoretical mass of the glycoform. Using tandem mass spectrometry in the negative ion mode, the topology of the glycoform was determined. It was found that the three hexoses were branched off of Hep[I] in a linear fashion and that Hep[II] remained unbranched. The topology analysis was confirmed through use of STAT, which yielded two possible glycoforms in which the hexoses were branched or linear. Using mild acid hydrolysis, the linkage position of the penultimate hexose to the terminating hexose was found to be $1 \rightarrow 4$. This linkage position was confirmed through comparison of the CID spectrum of the hydrolysis product to that of a maltose standard. Using the combined MS data, the structure of the LOS from N. gonorrhoeae strain 302 was found to be:

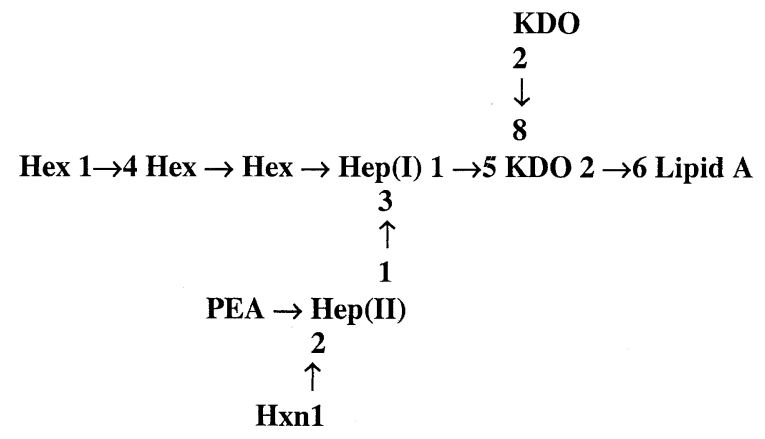

\section{Acknowledgments}

JAL and MDL gratefully acknowledge NIH grant (GM 47356) for funding. RY gratefully acknowledges funding from Grants-in-Aid for scientific research, from the Ministry of Education (10306022) for Science, Sports, and Culture, Japan.

\section{References}

1. World Health Organization Web Page (http://www.who.ch.), fact sheet N 110 . 
2. CDC. Sexually Transmitted Diseases Surveillance Supplement: Gonococcal Isolate Surveillance Project (GISP) Annual Report, 1999. U.S. Department of Health and Human Services, Public Health Service: Atlanta, GA, October 2000.

3. Yamasaki, R.; Koshino, H.; Kurono, S.; Nishinaka, Y.; McQuillen, D. P.; Kume, A.; Gulati, S.; Rice, P. A. J. Biol. Chem. 1999, 274, 36550-36558.

4. John, C. M.; Grifiss, J. M.; Apicella, M. A.; Mandrell, R. E.; Gibson, B. W. J. Biol. Chem. 1991, 266, 19303-19311.

5. Yamasaki, R.; Kenwood, D. E.; Schneider, H.; Quinn, K. P.; Griffiss, J. M.; Mandrell, R. E. J. Biol. Chem. 1994, 269, 3034530351.

6. Preston, A.; Mandrell, R. E.; Gibson, B. W.; Apicella, M. A. Crit. Rev. Microbiol. 1996, 22, 139-180.

7. Harvey, D. J. Mass Spectrom. Rev. 1999, 18, 349-450.

8. Muhlecker, W.; Gulati, S.; McQuillen, D. P.; Ram, S.; Rice, P. A.; Reinhold, V. N. Glycobiol. 1999, 9, 157-171.

9. Xie, Y.; Tseng, K.; Lebrilla, C. B.; Hedrick, J. L. J. Am. Soc. Mass Spectrom. 2001, 12, 877-884.

10. Gaucher, S. P.; Cancilla, M. T.; Phillips, N. J.; Gibson, B. W.; Leary, J. A. Biochem. 2000, 39, 12406-12414.

11. Zhou, Z.; Ogden, S.; Leary, J. A. J. Org. Chem. 1990, 55, $5444-5446$.
12. Hofmeister, G. E.; Zhou, Z.; Leary, J. A. J. Am. Chem. Soc. 1991, 113, 5964-5970.

13. Fura, A.; Leary, J. A. Anal. Chem. 1993, 65, 2805-2811.

14. Sible, E. M.; Brimmer, S. P.; Leary, J. A. J. Am. Mass Soc. Spectrom. 1997, 8, 32-42.

15. König, S.; Leary, J. A. J. Am. Soc. Mass Spectrom. 1998, 9, 1125-1134.

16. Cancilla, M. T.; Gaugher, S. P.; Desaire, H.; Leary, J. A. Anal. Chem. 2000, 72, 2901-2907.

17. Gaugher, S. P.; Morrow, J.; Leary, J. A. Anal. Chem. 2000, 72, 2331-2336.

18. Asam, M. R.; Glish, G. L. Anal. Chem. 1997, 8, 987-995.

19. Westphal, O.; Jahn, K. Methods in Carbohydrate Chemistry; In: Whistler, R. L.; Wolfrom, M. L., Eds.; Academic Press: New York, 1965; p. 83-91.

20. McLaugh, W.; Engstrom, J. J.; Auriola, S.; Phillips, N. J.; Gibson, B. W. Mass Spectrometry as a Tool to Determine the Structure and Heterogeneity of Bacterial Lipooligosaccharides (LOS). Mass Spectrometry in the Biological Sciences; In: Carr, S. A.; Burlingame, A. L.; Totowa, N. J., Eds.; Humana Press, 1996, p. 403-424.

21. McLafferty, F. W.; Tureček, F. Interpretation of Mass Spectra; 4th ed. University Science Books: Mill Valley, 1993, pp 37-38. 